Southern Illinois University Edwardsville SPARK

SIUE Faculty Research, Scholarship, and Creative Activity

$8-12-2016$

\title{
Continuous Isolation of Monocytes using a Magnetophoretic-based Microfluidic Chip
}

Jeff Darabi

jdarabi@siue.edu

Chuan Guo

cguo@siue.edu

Follow this and additional works at: http://spark.siue.edu/siue_fac

Part of the Biomechanics and Biotransport Commons, and the Biomedical Devices and Instrumentation Commons

\section{Recommended Citation}

Darabi, Jeff and Guo, Chuan, "Continuous Isolation of Monocytes using a Magnetophoretic-based Microfluidic Chip" (2016). SIUE Faculty Research, Scholarship, and Creative Activity. 51.

http://spark.siue.edu/siue_fac/51 


\section{Cover Page Footnote}

This is an author-created, un-copyedited version of an article published in Biomedical Microdevices. The final publication is available online from Springer at DOI 10.1007/s10544-016-0105-8. 
This is an author-created, un-copyedited version of an article published in Biomedical Microdevices. The final publication is available online at DOI 10.1007/s10544-016-0105-8

\title{
Continuous Isolation of Monocytes using a Magnetophoretic-based Microfluidic Chip
}

\author{
Jeff Darabi* and Chuan Guo \\ Department of Mechanical Engineering \\ Southern Illinois University Edwardsville
}

Edwardsville, IL 62026

*Corresponding author, Phone: 618-650-3382; Email: jdarabi@siue.edu

\begin{abstract}
Monocytes play an important role in the immune system and are responsible for phagocytizing and degrading foreign microorganisms in the body. The isolation of monocytes is important in various immunological applications such as in-vitro culture of dendritic cells. We present a magnetophoretic-based microfluidic chip for rapid isolation of highly purified, untouched monocytes from human blood by a negative selection method. This bioseparation platform integrates several unique features into a microfluidic device, including locally engineered magnetic field gradients and a continuous flow with a buffer switching scheme to improve the performance of the cell separation process. The results indicate high monocyte purity and recovery performances at a volumetric flow rate that is nearly an order of magnitude larger than comparable microfluidic devices reported in literature. In addition, a comprehensive 2-D computational modeling is performed to determine the cell trajectory and trapping length within the microfluidic chip. Furthermore, the effects of channel height, substrate thickness, cell size, number of beads per cell, and sample flow rate on the cell separation performance are studied.
\end{abstract}


This is an author-created, un-copyedited version of an article published in Biomedical Microdevices. The final publication is available online at DOI 10.1007/s10544-016-0105-8

\section{Introduction}

Human monocytes are involved in innate immune system and are responsible for the host defense against pathogenic invaders such as viruses, bacteria, and parasites. They can differentiate into different cell types such as macrophages and dendritic cells to provoke and regulate an immune response and destroy pathogens or pathogen infected host cells (Yuan et al. 2007). The isolation of highly purified monocytes is important in immunological research such as in-vitro generation of dendritic cells from peripheral blood and to study infectious diseases. Over the past decade, several microfluidic-based concepts have been investigated to isolate cells from blood (Nagrath et al. 2007; Qu et al. 2008; Talasaz et al. 2009; Stott et al. 2010; Mach and Di Carlo 2010; Hoshino at al. 2011;Moon et al. 2011; Wu et al. 2013; Ozkumur et al. 2013; Karabacak et al. 2014). In recent years, several ferromagnetic-based microfluidic devices have been developed to isolate circulating tumor cells (CTCs) from human peripheral blood (S. Kim et al. 2013; Y.Y. Huang et al. 2015; W. Park et al. 2015). A capture efficiency of approximately $90 \%$ was demonstrated by spiking CTCs into human peripheral blood. However, these devices are generally hampered by complex fabrication processes and low volumetric throughputs. For example, the volumetric throughputs in some of these studies were limited to $0.48 \mathrm{~mL} / \mathrm{hr}$ (Stott et al. 2010) and less than $2.5 \mathrm{~mL} / \mathrm{h}$ (Wu et al. 2013). At these flow rates, the time required to isolate monocytes from a $10 \mathrm{~mL}$ sample is more than 4 hours. Such long processing times make the low-flow rate platforms unsuitable for clinical applications. To address some of these issues, we present a magnetophoretic microfluidic device for rapid isolation of highly purified, untouched monocytes from human blood by a negative selection method. Magnetophoretic separation is a process in which a magnetically labeled bioparticle is isolated from a mixture by applying a magnetic field. The process involves the labeling of the target bioparticles with antibodies and magnetic beads, followed by separating the tagged bioparticles via a magnetic separation device. The antibodies specifically bind to their matching antigen on the surface of the bioparticle and functionalized groups on the bead surface. Magnetophoretic separation can be implemented by a direct or an indirect method. In the direct method, surface-treated magnetic beads are first mixed with a sample containing the target bioparticles and incubated for a sufficient amount of time, where the functionalized magnetic beads bind to the bioparticles. The labeled bioparticles are then isolated from the solution by applying an external magnetic field. In the indirect approach, the target bioparticle is first tagged with a primary antibody. Magnetic beads with surface-bound secondary antibodies are then added to the mixture and incubated where magnetic beads bind to the primary antibody on the surface of the bioparticle. Additionally, the magnetophoretic separation can be either 
This is an author-created, un-copyedited version of an article published in Biomedical Microdevices. The final publication is available online at DOI 10.1007/s10544-016-0105-8

positive or negative. In positive selection, the magnetically labeled target cells are retained in the magnetic separation device, while the non-target particles flow through the device. In negative selection, however, the unwanted cells are retained and the untouched target cells flow through the chip and collected at an outlet of the bioseparation chip. The negative selection method is preferred in applications where alteration in functional properties of isolated cells is not desired.

Magnetophoretic separation has proven to be effective for the isolation of various types of bioparticles from their native environment (Zborowski et al. 1995). It is significantly faster than competing techniques and can isolate the target bioparticles directly from the crude samples such as blood, bone marrow, and tissue homogenates. In addition, since the unlabeled bioparticles have a very low magnetic susceptibility, the contrast between the labeled and unlabeled bioparticles is quite large, resulting in a high selectivity. The magnitude of the magnetic flux gradient is an important factor that affects the capture and isolation of magnetic particles. Both permanent magnets and electromagnets (Furlani and Sahoo 2006; Shevkoplyas et al. 2007) can be used to produce a magnetic flux gradient. However, electromagnets can generate a significant ohmic heating that may damage cells and decease cell viability.

In this paper, a negative selection method is used to isolate highly purified, untouched monocytes from human blood using a magnetophoretic-based microfluidic chip. Additionally, a two-dimensional computational modeling is performed to optimize the chip design and study the effects of channel height, substrate thickness, cell size, number of beads per cell, and sample flow rate on cell separation performance.

\section{Materials and Methods}

\subsection{Fabrication and Operation of Bioseparation Chip}

A schematic of the microfluidic-based magnetophoretic bioseparation chip is shown in Fig. 1. A sample containing mononuclear cells is first mixed and incubated with a cocktail of biotinylated monoclonal antibodies to magnetically label non-monocytes. The sample is then introduced into the chip, where non-monocytes are depleted by retaining them in the microfluidic chip, while the untouched monocytes flow through the chip. A continuous buffer flow prevents cells from sticking to the channel wall and also improves cell purity by focusing the sample to the center region of the channel. This bioseparation platform incorporates several unique features into a microfluidic device, including the use of short- and long-range magnetic 
This is an author-created, un-copyedited version of an article published in Biomedical Microdevices. The final publication is available online at DOI 10.1007/s10544-016-0105-8

field gradients, and a continuous flow with a buffer switching scheme to improve the performance of the cell separation process (Darabi and Guo 2013). The direction and magnitude of the force on a magnetically-labeled target particle are governed by the gradient of the magnetic flux. Thus, in order to achieve an efficient and reproducible magnetophoretic separation, the precise control of this parameter in the vicinity of the magnetic particle is crucial. In our design, a thin nickel grid is deposited and patterned on a glass substrate to precisely control the magnetic field gradients. Since nickel has a much higher permeability compared to its surroundings, a strong magnetic field gradient is created at the edges of the nickel grid when it is subjected to an external magnetic field. In addition, an array of external magnets with opposing poles is placed on the bottom side of the chip. This arrangement creates a large, long-range non-uniform magnetic field at the edges and interface of the magnets to selectively pulled down magnetically labeled cells from the stream and immobilize them on the nickel grids.

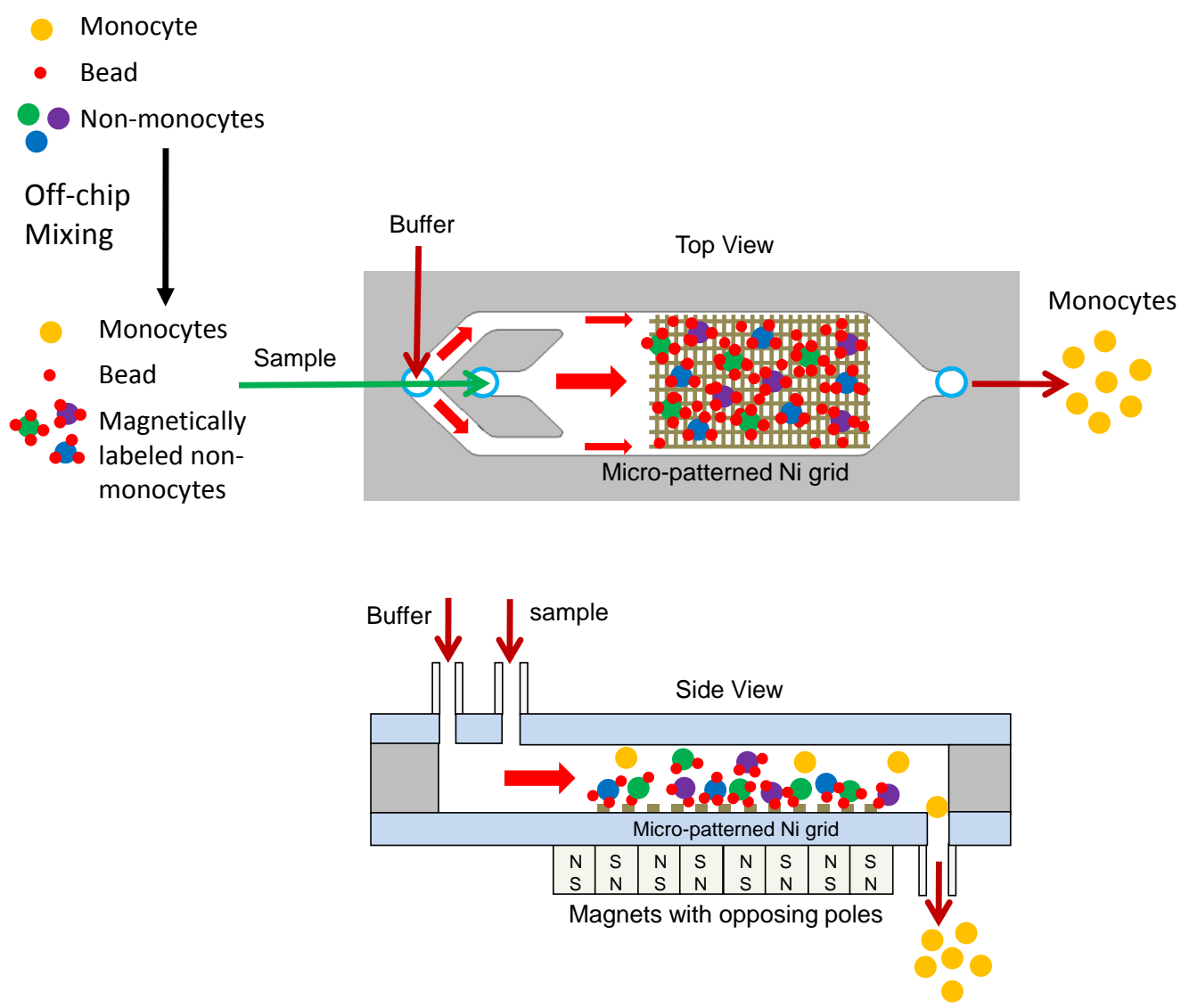

Figure 1 Schematic illustration of a monocyte isolation chip. A sample containing untouched monocytes and non-target cell-bead complexes are introduced into the chip. The non-target cell-bead complexes are selectively pulled down and trapped at the edges of the nickel strips while the untouched monocytes flow through the chip to a collection tube. 
This is an author-created, un-copyedited version of an article published in Biomedical Microdevices. The final publication is available online at DOI 10.1007/s10544-016-0105-8

The chip consists of a glass substrate on which a nickel grid is deposited and patterned. The overall dimensions of the chip are approximately $25 \mathrm{~mm} \times 75 \mathrm{~mm} \times 1 \mathrm{~mm}$. The chip has a fluidic channel height of $100 \mu \mathrm{m}$, a nickel width of $10 \mu \mathrm{m}$, a nickel spacing of $25 \mu \mathrm{m}$, and a nickel thickness of $0.2 \mu \mathrm{m}$. The fluidic ports were drilled into another glass substrate. The glass substrates were then cleaned and bonded using a double-sided polyimide film. More details on the chip fabrication and assembly can be found in an earlier publication of the authors (Darabi and Guo 2013). The experimental setup consists of a microfluidic chip, a bidirectional MilliGAT pump with a MicroLynx controller for the sample, a syringe pump for the buffer, and plastic tubing for making fluidic connections between the pumps and the chip. The MilliGAT pump is a programmable bidirectional pump which is capable of infusing and withdrawing. The fluid withdrawal feature is ideal for microfluidic applications where smaller sample volumes are used. To characterize the performance of the device, the chip was secured on a microscope stage to view the cell motion and capture within the fluidic channel as shown in Fig. 2.

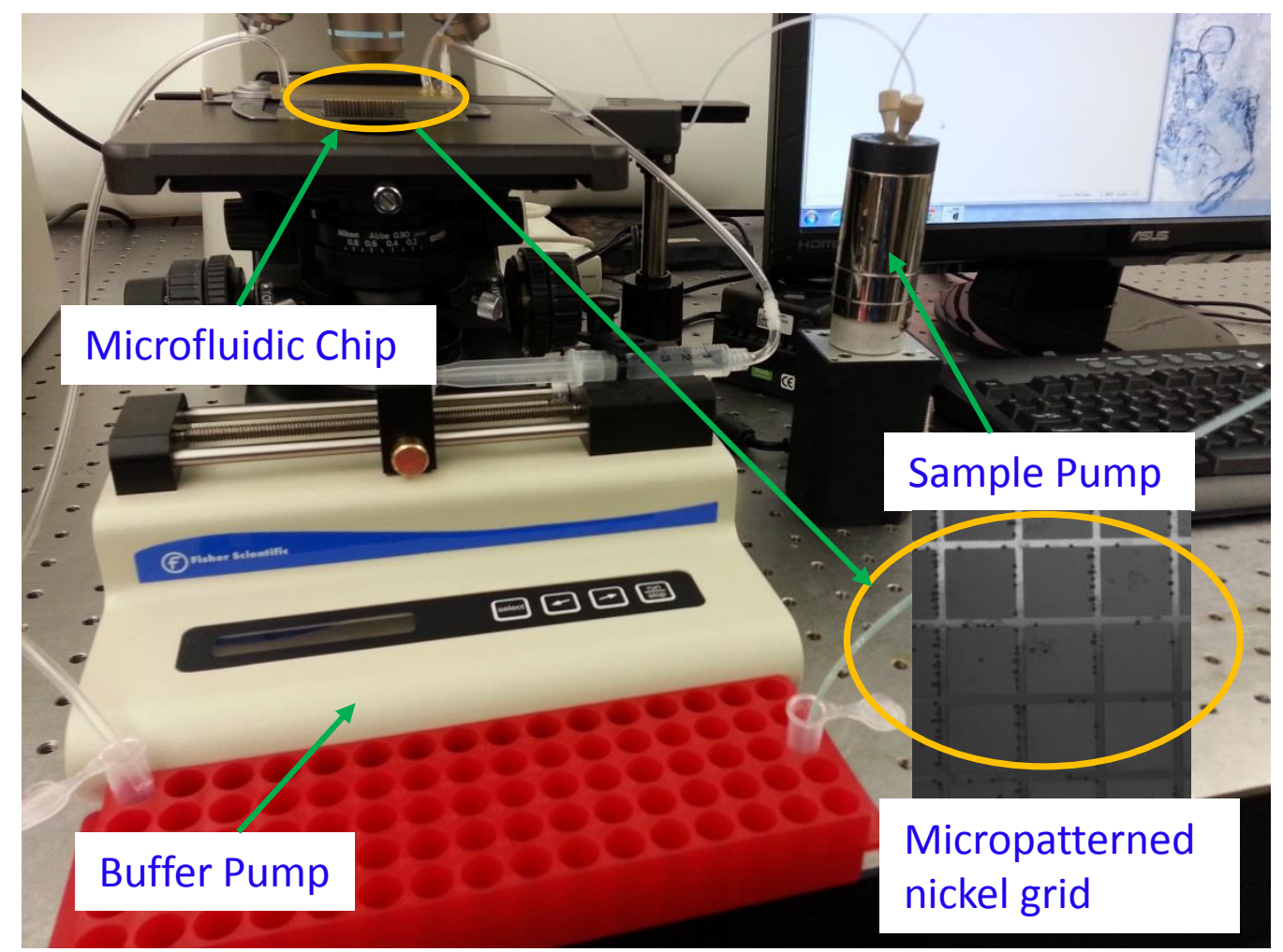

Figure 2 A photograph of the test setup. The insert in the bottom right corner shows a micrograph of the micropatterned nickel grid with trapped cells. 
This is an author-created, un-copyedited version of an article published in Biomedical Microdevices. The final publication is available online at DOI 10.1007/s10544-016-0105-8

\subsection{Monocyte Isolation Experiments}

In this work, untouched monocytes were isolated by depletion of non-monocytes from human blood using a Monocyte Isolation Kit (Life Technologies, Carlsbad, CA). Peripheral blood mononuclear cells (MNCs) were first obtained from fresh human blood by the Ficoll-Paque PLUS method. Approximately $10^{7}$ MNCs were transferred to a 1.5-mL Eppendorf tube and incubated with $20 \mu \mathrm{L}$ blocking reagent and $20 \mu \mathrm{L}$ antibody mix (Life Technologies). To isolate pure monocytes from mononuclear cells by negative isolation, an antibody mix containing biotinylated mouse IgG antibodies for CD3, CD7, CD16, CD19, CD56, CDw123 and CD235a (Life Technologies) was added to the tube. This antibody mix binds to non-monocytes (i.e T cells, B cells, NK cells, dendritic cells, erythrocytes, granulocytes, and macrophages). The sample was mixed well and incubated with gentle rotation at $2-8^{\circ} \mathrm{C}$ for 20 minutes. The sample was centrifuged at $300 \mathrm{~g}$ for 8 minutes at $2-8^{\circ} \mathrm{C}$ and supernatant was removed. The pellets were re-suspended in $900 \mu \mathrm{L}$ cold isolation buffer. $100 \mu \mathrm{L}$ pre-washed MyOne Dynabeads were added to the sample and mixed well by pipetting to attach the antibody-labeled non-target cells to the Dynabeads. The sample was incubated for 15 minutes at $2-8^{\circ} \mathrm{C}$ with gentle rotation.

Prior to each monocyte isolation test, the chip and tubing were washed with $10 \%$ bleach and rinsed with deionized water. It was then soaked with $20 \%$ bovine serum albumin (BSA) for 30 min and rinsed with isolation buffer. Next, the sample was withdrawn into the tubing using the bidirectional milliGAT pump and the sample tubing was connected to the sample inlet port. The sample containing untouched target monocytes and magnetically-labeled non-monocytes was introduced into the chip. The buffer flow rate was set to $5 \mathrm{~mL} / \mathrm{h}$ using the syringe pump. The sample flow rate was varied from $10 \mathrm{~mL} / \mathrm{hr}$ to $50 \mathrm{~mL} / \mathrm{hr}$ in $10 \mathrm{~mL} / \mathrm{hr}$ increments. When the sample is introduced into the chip, bead bound cells (i.e. T cells, B cells, NK cells, dendritic cells, granulocytes, and erythrocytes) and free beads are selectively pulled down and trapped at the edges of the nickel grid in the magnetic trapping region, while the untouched human monocytes flow through the chip and are collected at an outlet port of the chip. Once, the entire sample was infused, the buffer flow rate was increased to $20 \mathrm{~mL} / \mathrm{hr}$ and kept running until the monocyte collection tube reached $1 \mathrm{~mL}$. The collected human monocytes can be then used in any application such as cell culture, generation of monocyte derived dendritic cells, functional assays, molecular studies and flow cytometry. After each test, the bead-bound cells and free beads are eluted form the chip and discarded. 
This is an author-created, un-copyedited version of an article published in Biomedical Microdevices. The final publication is available online at DOI 10.1007/s10544-016-0105-8

To obtain monocyte purity and recovery, the isolated monocyte samples and pre-isolation samples were individually stained with $10 \mu \mathrm{L}$ of CD45 FITC/CD14-PE antibody solution (BD Biosciences) and analyzed on a flow cytometer. The pre-isolation isotype control sample was stained with a $20 \mu \mathrm{L}$ IgG1 FITC/IgG1 PE antibody and incubated at room temperature with gentle rotation for 30 minutes. The samples were centrifuged at $350 \mathrm{~g}$ for 8 minutes and supernatants were carefully removed. The pellets were re-suspended in $200 \mu \mathrm{L}$ isolation buffer and analyzed on a FACS Aria (BD Biosciences). Each test was repeated three times and the average values and standard deviations of monocyte purity and recovery were calculated.

Figure 3 shows a representative flow cytometric dot plot of the sample before and after monocyte isolation. Monocytes are identified as positive for both CD45 FITC/CD14-PE, as represented by the Q2 quadrant on the dot plot. Regions Q1, Q3, and Q4 represent non-target cell populations. The concentration of the monocytes prior to isolation was $13.6 \%$. After isolation, the purity of the monocytes increased to $86.4 \%$. Figure 4 shows percent purity and recovery of the monocytes as a function of the sample flow rates. The sample flow rate was varied from $10 \mathrm{~mL} / \mathrm{hr}$ to $50 \mathrm{~mL} / \mathrm{hr}$. The results indicate that the cell purity decreases with increasing the sample flow rate while the cell recovery increases. This is due to the fact that the hydrodynamic force increases with increasing the sample flow rate. Thus, the magnetophoretic force is not sufficient enough to pull down and trap magnetically-labeled nonmonocytes with fewer numbers of beads per cell. As a result, some of the non-monocytes flow through channel into the collection tube, resulting in lower cell purity. However, as the flow rate increases, the likelihood of none-specific monocyte trapping under magnetically-labeled non-target cells decreases as well, resulting in a higher recovery performance. 
This is an author-created, un-copyedited version of an article published in Biomedical Microdevices. The final publication is available online at DOI 10.1007/s10544-016-0105-8

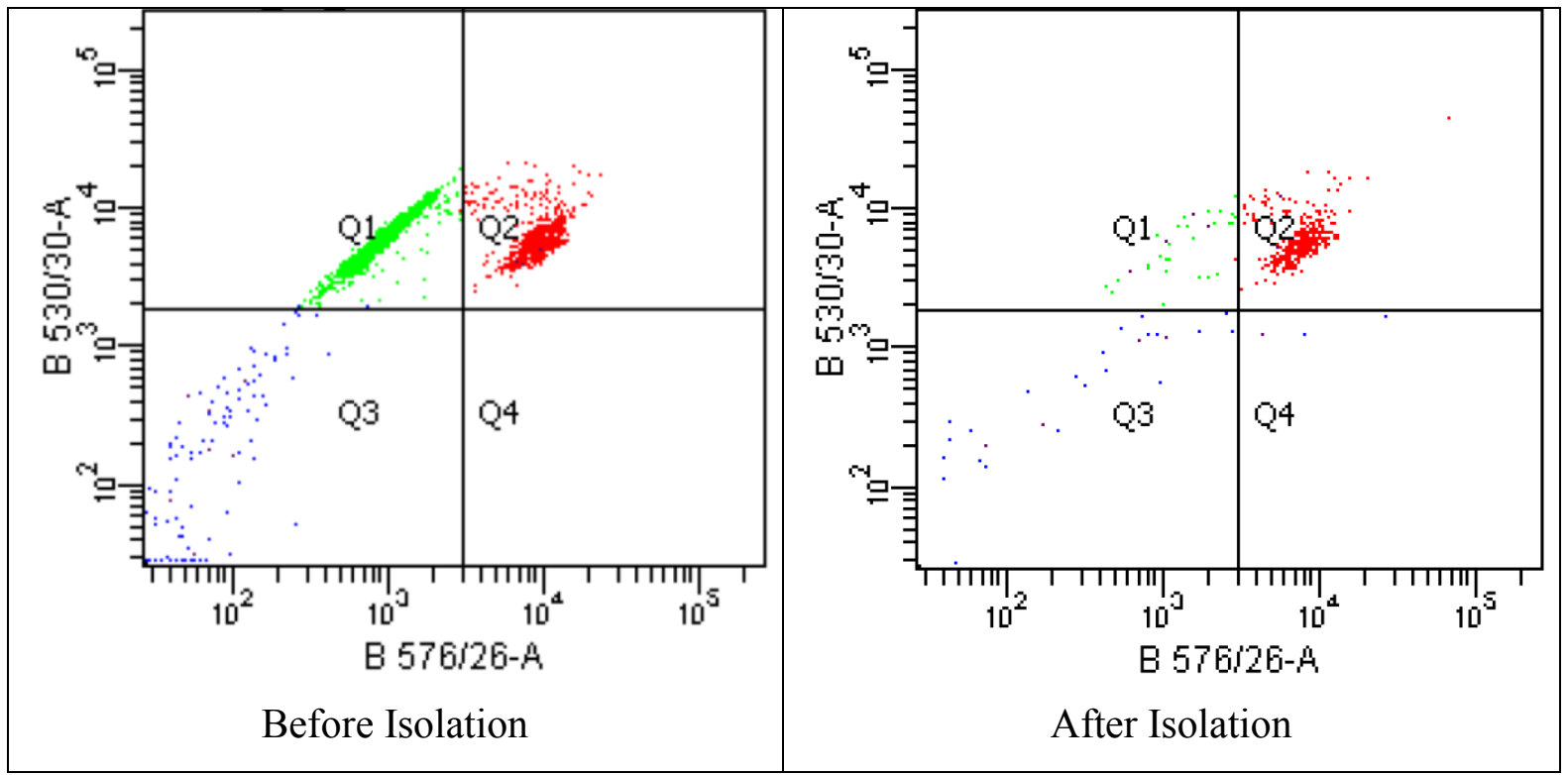

Figure 3 Flow cytometric analyses before and after monocyte isolation. Quadrant regions show the percentage of cells in each sub-population. Monocytes are identified as positive for both CD45 FITC and CD14-PE, as represented by the Q2 quadrant on the dot plot. The concentration of the monocytes was $13.6 \%$ prior to isolation. After isolation, the purity of the monocytes increased to $86.4 \%$.

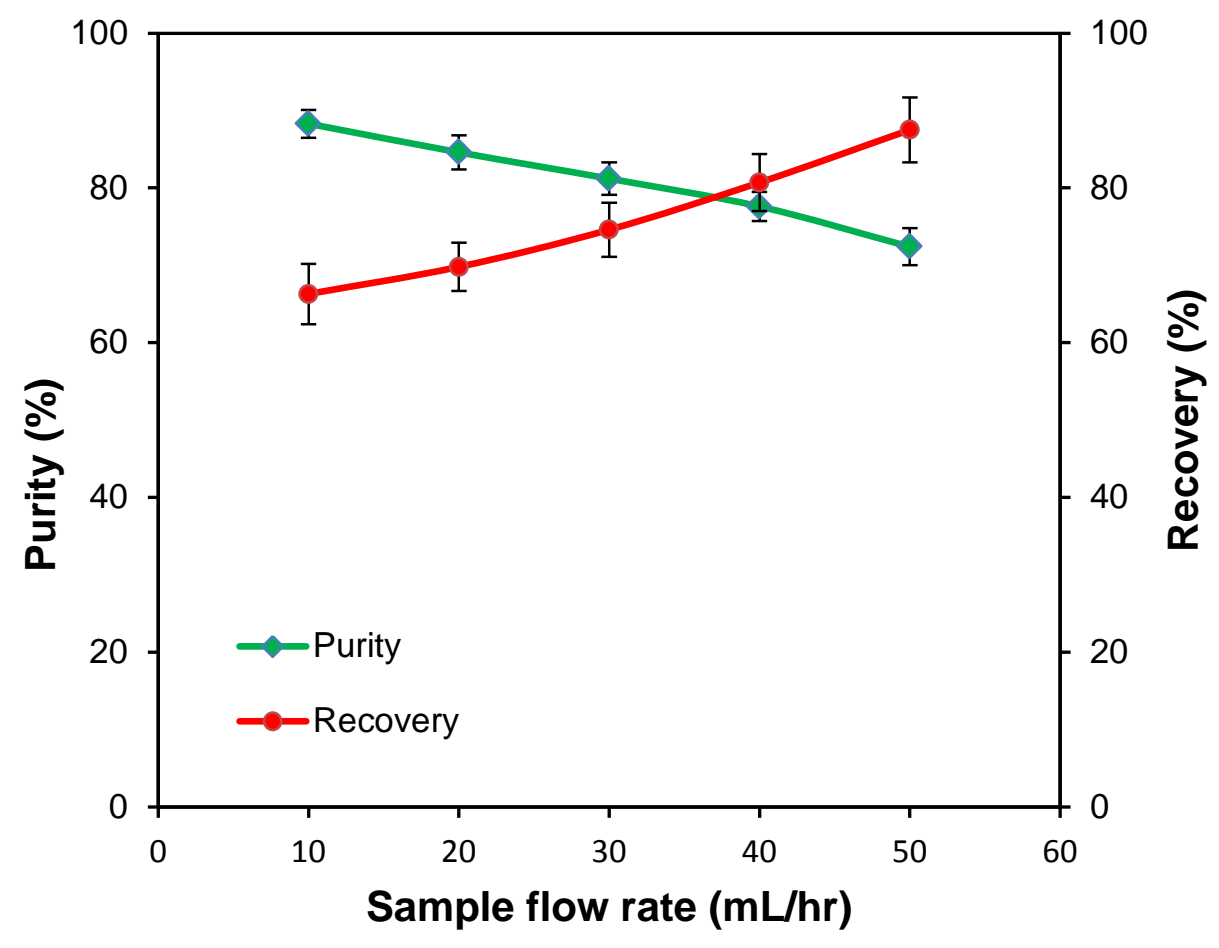

Figure 4 Purity and recovery of isolated monocytes by negative depletion method at various sample flow rates. The error bars depict the standard deviation. 
This is an author-created, un-copyedited version of an article published in Biomedical Microdevices. The final publication is available online at DOI 10.1007/s10544-016-0105-8

\section{Modeling and Simulation}

\subsection{Simulation of Long-range Permanent Magnets}

The AC/DC module in COMSOL Multiphysics was used to simulate magnetic field gradients surrounding an array of permanent magnets. The permanent magnets used in this study were grade N52 NdFeB magnets (K\&J Magnetics) with dimensions of $19 \mathrm{~mm} \times 1.6 \mathrm{~mm} \times 6.35 \mathrm{~mm}$ and were magnetized through the $6.35 \mathrm{~mm}$ dimension. These magnets are made from an alloy containing Neodymium, Iron, and Boron. The relative permeability and remanence magnetization of the magnets are 1.05, and $1.43 \mathrm{~T}$, respectively (Campbell 1996). Initially, the magnetic field simulations were performed for a single magnet to verify our modeling approach. The magnetic flux generated by a permanent magnet is given by

$$
\vec{B}=\mu(\vec{H}+\vec{M})
$$

where $\vec{B}$ is the magnetic flux density, $\vec{H}$ is the magnetic field strength, $\vec{M}$ is the remanence magnetization, and $\mu$ is the magnetic permeability. When the Magnetic Fields with no currents is selected in COMSOL, the model solves the magnetic scalar potential, $\mathrm{V}_{\mathrm{m}}$, given by

$$
\nabla \cdot \mu\left(-\nabla V_{m}+\overrightarrow{M_{0}}\right)=0
$$

The magnetic flux density can be then obtained from the magnetic scalar potential using:

$$
\vec{B}=\mu\left(-\nabla V_{m}+\overrightarrow{M_{0}}\right)
$$

where $\overrightarrow{M_{0}}$ is the initial remanence magnetization. To verify our magnetic field simulations results for a single magnet, the magnetic flux at the center of the magnet was compared with the technical data reported by K\&J magnetics. The simulated and reported magnetic flux density at the surface of the magnet were $0.70 \mathrm{~T}$ and $0.69 \mathrm{~T}$, respectively, validating our modeling results.

Once a single magnet was simulated and verified, simulations were performed for an array of permanent magnets with opposing poles to obtain long-range magnetic field gradients. The number of magnets was varied from 2 to 14 . The simulations results revealed that when 8 or more magnets were used, the fringing effect at the ends of the array of magnets was minimal and the magnitude of the magnetic field was nearly the same at each interface. Normal magnetic fluxes at various distances from the surface of the magnets for an array of 10 magnets is shown in Fig. 5. All dimensions are in mm. The results clearly show that the magnetic flux reaches 
This is an author-created, un-copyedited version of an article published in Biomedical Microdevices. The final publication is available online at DOI 10.1007/s10544-016-0105-8

peak values at the interface between adjacent magnets. In addition, the results show that the peak values of the magnetic flux decrease as the distance from the surface of the magnets increases. In the rest of the simulation study, the magnetic flux density along the channel was assumed to be a repetition of the magnetic flux between two peaks.

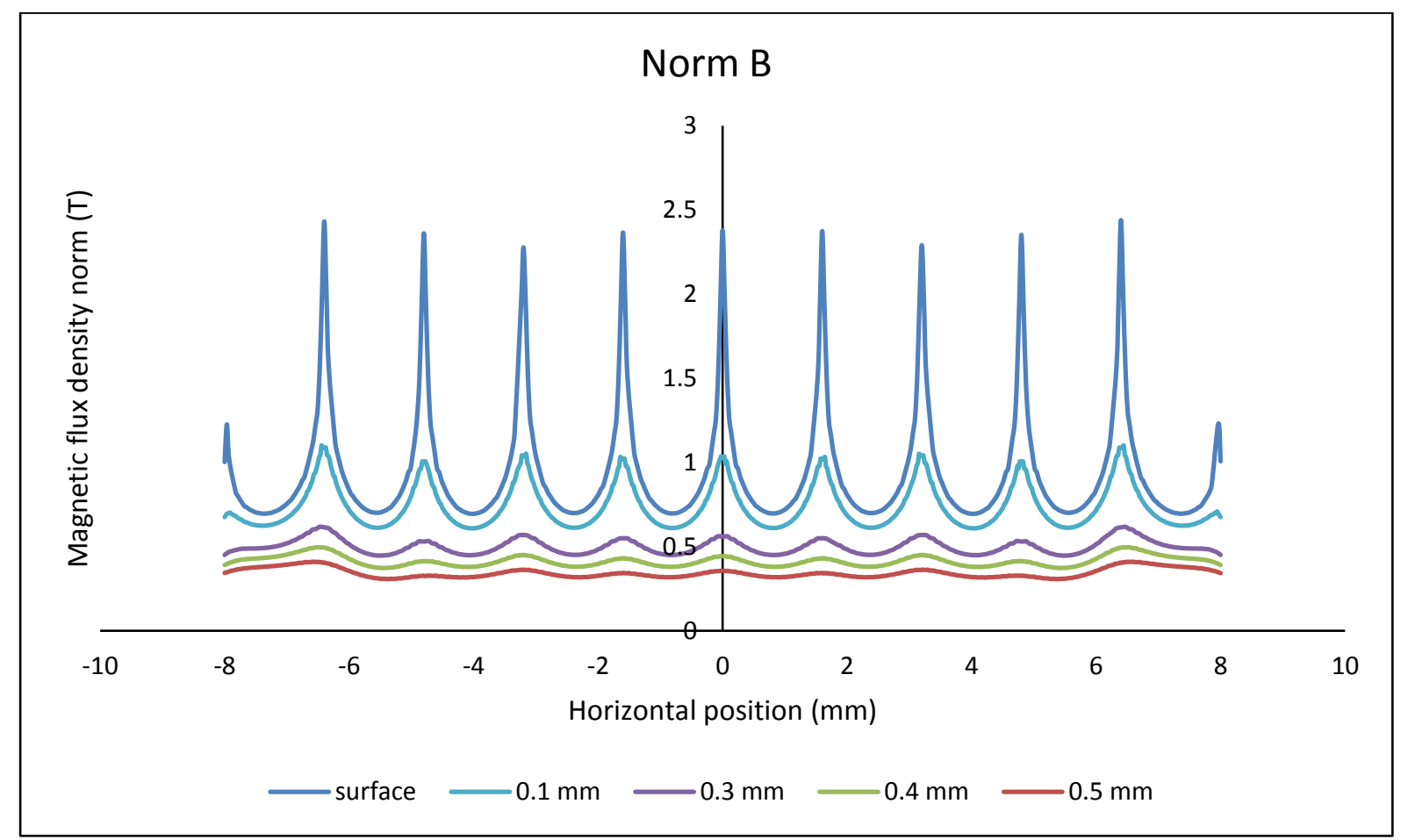

Figure 5 Normal magnetic flux density for an array of 10 magnets at various distances from the surface of the magnets

\subsection{Simulation of Short-range Micro-scale Gradients}

One of the key features of our device is the use of short-range magnetic field gradients, generated by an array of micro-patterned nickel grids on the bottom substrate (Hale and Darabi 2014). The purpose of the nickel grid is to create high microscale magnetic field gradients to trap and immobilize magnetic particles, and enable uniform distribution of the captured cells. Figure 6 depicts the effect of micro-patterned nickel grid on the short-range magnetic field gradients. The width and thickness of each strip were $10 \mu \mathrm{m}$ and $0.2 \mu \mathrm{m}$, respectively. The gap between the strips was $25 \mu \mathrm{m}$. Periodic boundary conditions were applied to the left and right sides of the computational domain. A uniform magnetic flux boundary condition is applied to the bottom surface. The simulation results show that the magnetic field strength increases by up to eight-fold at the edges of the nickel grids. 


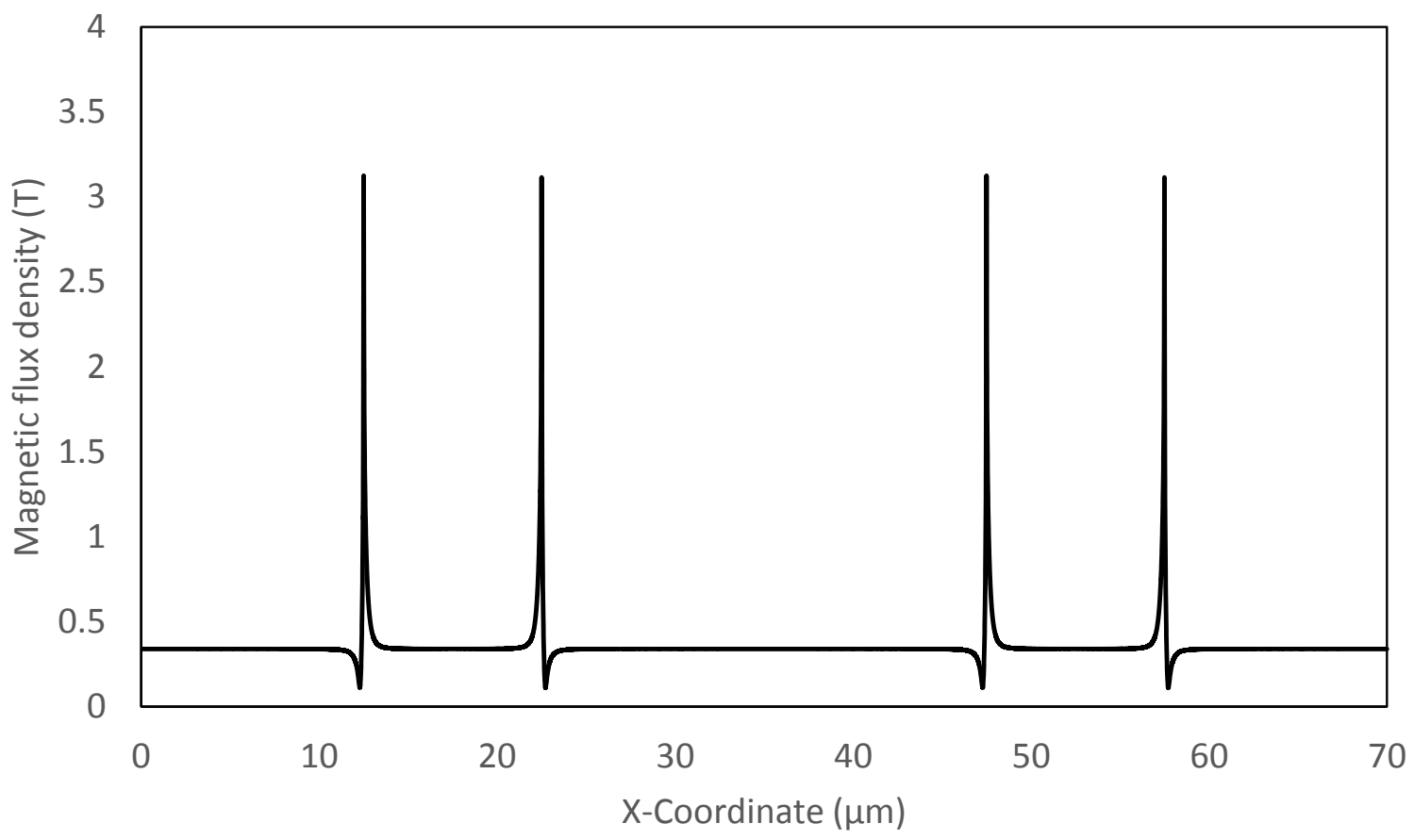

Figure 6 Magnetic field simulations above a pair of nickel strips. The magnetic flux density increases by up to eight-fold at the edges of the nickel grids.

\subsection{Simulation of Particle Trajectory and Motion}

A 2-D model was developed in a Cartesian coordinates to simulate the particle trajectory and trapping along the fluidic channel. Figure 7 shows a schematic diagram of the computational domain. This 2-D representation is adequate due to a large aspect ratio of the width of the microfluidic channel to its height (which was 100:1 in this study). Only a single pair of nickel strips was included in the model. The presence of a large number of neighboring nickel strips was simulated by imposing periodic boundary conditions on the left and right sides of the model. COMSOL Multiphysics was used to perform two-dimensional numerical simulations of the magnetic fields above the nickel strips. The values of the magnetic field at discrete points within the model were then exported into MATLAB to compute the magnetic field gradient at discrete points within the model. The positions of these nodes and their corresponding magnetic field values were arranged and interpolated to create position and magnetic field gradient matrices. 
This is an author-created, un-copyedited version of an article published in Biomedical Microdevices. The final publication is available online at DOI 10.1007/s10544-016-0105-8

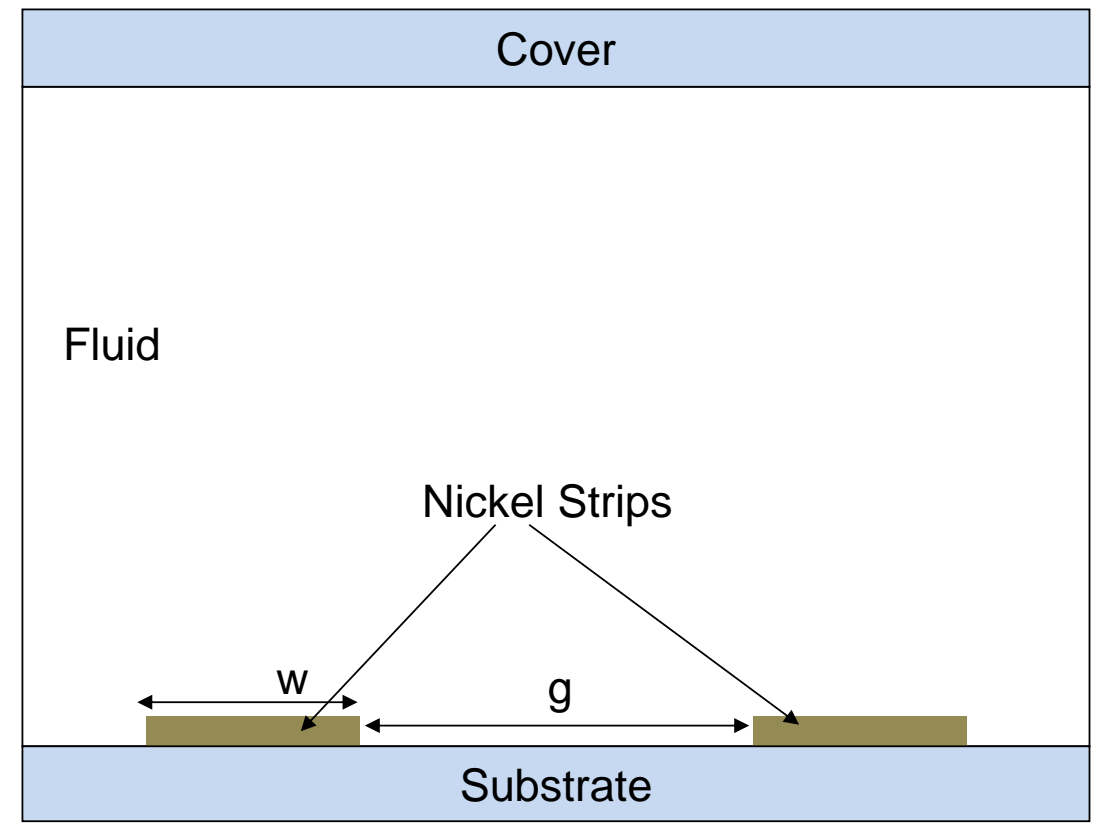

Figure 7 A schematic diagram of the 2-D computational domain. The thickness of the nickel film is not drawn to scale. $\mathrm{w}$ and $\mathrm{g}$ represent the nickel strips width and gap.

It is assumed that the particle under examination is spherical. Brownian forces and particle-toparticle interactions are assumed negligible. In the presence of an external magnetic field, the motion of a magnetophoretic particle is mainly governed by the magnetophoretic force, $\vec{F}_{m}$, the hydrodynamic drag force, $\vec{F}_{d}$, and to lesser extend the net gravitational force, $\vec{F}_{g}$. It should be noted that the forces due to gravity and buoyancy are included for the sake of generalization of the model. However, they are approximately an order of magnitude smaller than the other forces. The motion of a magnetic particle flowing through a fluidic channel can be predicted by Newton's second law

$$
m_{p} \frac{d \vec{v}_{p}}{d t}=\vec{F}_{m}+\vec{F}_{g}+\vec{F}_{d}
$$

where $m_{p}$ and $\vec{V}_{p}$ are the mass and velocity of the particle, respectively. The magnetophoretic force acting on a single magnetic bead is given by (Purcell 1985)

$$
\vec{F}_{m}=\left(\vec{m}_{b} \cdot \nabla\right) \vec{B}
$$

where $\vec{B}$ is the applied magnetic field, $\vec{m}_{b}$ is the magnetic moment of the magnetic bead, expressed by

$$
\vec{m}_{b}=V_{b} \vec{M}_{b}
$$


This is an author-created, un-copyedited version of an article published in Biomedical Microdevices. The final publication is available online at DOI 10.1007/s10544-016-0105-8

where $\vec{M}_{b}$ is the volumetric magnetization of the magnetic bead. For superparamagnetic beads used in this study, the hysteresis effect is negligible and magnetization curves overlap when magnetic field is increased or decreased (Darabi and Guo 2013). In addition, as depicted in Fig. 3 , the magnetic flux density generated within the fluidic channel is greater than $0.4 \mathrm{~T}$. Thus, the magnetization of superparamagnetic beads is saturated and the magnetic force acting on the particle can be found from:

$$
\vec{F}_{m}=V_{b} \vec{M}_{s a t} \cdot \nabla \vec{B}
$$

where $\vec{M}_{\text {sat }}$ is the volumetric saturation magnetization of the superparamagnetic beads. As discussed in the previous section, the magnetic field produced by an array of permanent magnets can be considerably complicated. Also, to implement an effective cell separation, several micron-sized magnetic beads must bind to the surface of the cell. Thus, a particle traveling through the fluidic channel is not spherical in shape. In addition, since the magnetic beads are randomly attached to the surface of the cell, the direction of the magnetic force on each bead is slightly different depending on the location of the beads. However, in many magnetophoretic-based cell separations, the target cell is much larger than a single magnetic bead. For example, the average size of mononuclear cells (lymphocytes and monocytes) varies between 8-12 $\mu \mathrm{m}$ while a $1 \mu \mathrm{m}$ bead size was used in this study. Also, the distance between the beads on a cell is much smaller than the distance between the cell and the permanent magnets. Thus, the labeled cell-beads complex can be considered as a sphere with an effective radius estimated from (Safaryk and Safarykova 1997),

$$
\mathrm{R}_{p}=\left(\frac{3}{4} V_{P} / \pi\right)^{1 / 3}
$$

where $\mathrm{R}_{p}$ is the effective radius of the cell-beads complex and $V_{p}$ is the total volume of the cell and magnetic beads. The total magnetophoretic force acting on a magnetically-labeled cell can be estimated from,

$$
\vec{F}_{m}=N V_{b} \vec{M}_{s a t} \cdot \nabla \vec{B}
$$

where $\mathrm{N}$ is the number magnetic beads bound to a cell. The hydrodynamic drag force refers to a force acting opposite to the direction of the movement. At low Reynold numbers, the drag force is mainly affected by the relative motion of the particle with respect to its surrounding medium and is expressed by Stokes' Law, 
This is an author-created, un-copyedited version of an article published in Biomedical Microdevices. The final publication is available online at DOI 10.1007/s10544-016-0105-8

$$
\vec{F}_{d}=6 \pi \mathrm{R}_{p} \eta\left(\vec{V}_{f}-\vec{V}_{p}\right)
$$

where $\vec{V}_{p}$ is the particle velocity, $R_{p}$ is the particle radius, $\vec{V}_{f}$ is the fluid velocity, and $\eta$ is the fluid viscosity. The net gravitational force is defined here as the difference between the downward gravitational and upward buoyancy forces and is given by

$$
\vec{F}_{g}=\left(\rho_{p}-\rho_{f}\right) v_{p} \vec{g}
$$

where $\rho_{p}$ is the particle density, $\rho_{f}$ is the fluid density, and $v_{p}$ is the particle volume. Since the density of the particle is larger than the density of the fluid, the particle experiences a net gravitational force toward the bottom of the channel, which may affect the particle trapping especially at low flow rates.

Force matrices were generated for the magnetophoretic force (Eq. 9), the hydrodynamic drag force (Eq. 10), and the net gravitation force (Eq. 11) within the microfluidic chip. The components of these three matrices were summed to generate matrices for the total force in both the axial (x) and the normal (y) directions. These force matrices were then used to calculate the axial and normal components of each cell's velocity relative to the fluid as follows:

In the $\mathrm{x}$-direction

$$
m_{p} \frac{d V_{p, x}}{d t}=F_{d, x}+F_{m, x}
$$

In the y-direction

$$
m_{p} \frac{d V_{p, y}}{d t}=F_{d, y}+F_{m, y}+F_{g}
$$

The velocity profile for a fully developed laminar flow in microfluidic channels is defined as:

$$
V_{f}=6 \frac{Q}{h w} \frac{y}{h}\left(1-\frac{y}{h}\right)
$$

where $Q$ is the volumetric flow rate, $h$ is the channel height, $w$ is the channel width, and $\mathrm{y}$ is the height within the channel at which the velocity is calculated. Therefore, the absolute velocity of the spherical particles in the microfluidic channel can be calculated as: 
This is an author-created, un-copyedited version of an article published in Biomedical Microdevices. The final publication is available online at DOI 10.1007/s10544-016-0105-8

$$
\begin{gathered}
V_{x}=V_{p, x}+6 \frac{Q}{h w} \frac{y}{h}\left(1-\frac{y}{h}\right) \\
V_{y}=V_{p, y}
\end{gathered}
$$

where $\mathrm{V}_{\mathrm{x}}$ and $\mathrm{V}_{\mathrm{y}}$ are the absolute particle velocities in the axial and normal directions. The next step after the velocities are obtained is to calculate the position of the particles with respect to time. This is done by assuming that the particles move at a constant velocity for a discreet amount of time. By defining the particle position, $S_{i}\left(x_{i}, y_{i}\right)$, the initial particle position at the channel entrance is set to $(0,0)$. As the time is incremented, a new position for each particle is obtained from the following equations:

$$
\begin{aligned}
& x_{i}=x_{i-1}+\left(V_{x}\right)_{i-1} \Delta t \\
& y_{i}=y_{i-1}+\left(V_{y}\right)_{i-1} \Delta t
\end{aligned}
$$

where $\mathrm{x}$ and $\mathrm{y}$ refer to the horizontal and vertical position of the particle, and $\Delta t$ is the timestep. Next, the forces acting on the particle at the new position were obtained and the velocities of the particle were calculated. By repeating these steps, the trajectory of each particle with respect to time was determined until it is captured on the bottom channel or it flows through the channel. The particle is considered captured when

$$
y_{n}=h
$$

Once the above condition is met, the trapping length and time are found from

$$
\begin{aligned}
& L=x_{n} \\
& \mathrm{~T}=\mathrm{n} \Delta \mathrm{t}
\end{aligned}
$$

\subsection{Modeling Results}

Since it is not possible to simulate purity and recovery data, a key parameter by which the performance of the bioseparation chip was evaluated in this study was the trapping length, which refers to a horizontal distance that a particle travels along the separation channel before it is trapped on the bottom of the channel. To ensure that most of the magnetically labeled cells can be trapped within the microfluidic channel, the trapping length and time were calculated for an extreme case where the particles travel along the top surface of the channel. To validate 
This is an author-created, un-copyedited version of an article published in Biomedical Microdevices. The final publication is available online at DOI 10.1007/s10544-016-0105-8

the computational model, a series of experiments were carried out using $1-\mu \mathrm{m}$ superparamagnetic beads and the simulated trapping lengths were compared with the experimentally measured trapping lengths. The sample flow rate was varied from $10 \mathrm{~mL} / \mathrm{h}$ to $50 \mathrm{~mL} / \mathrm{h}$ in $10 \mathrm{~mL} / \mathrm{h}$ increments. The buffer flow rate was set to $5 \mathrm{~mL} / \mathrm{h}$ during all bead capture experiments. Each test was repeated three times and the average values of the trapping length of superparamagnetic beads were calculated. The trapping length indicates the farthest distance the magnetic beads can travel before being captured. Figure 8 shows a comparison between the experimental and simulation results. It can be seen from these results that a good agreement is obtained between the experimental and simulation results. Once validated, the model was used to optimize the chip design and to study the effects of geometric and operating parameters such as number of magnetic bead per cell, cell size, channel height, substrate thickness, and flow rate on the cell trajectory along the microfluidic channel.

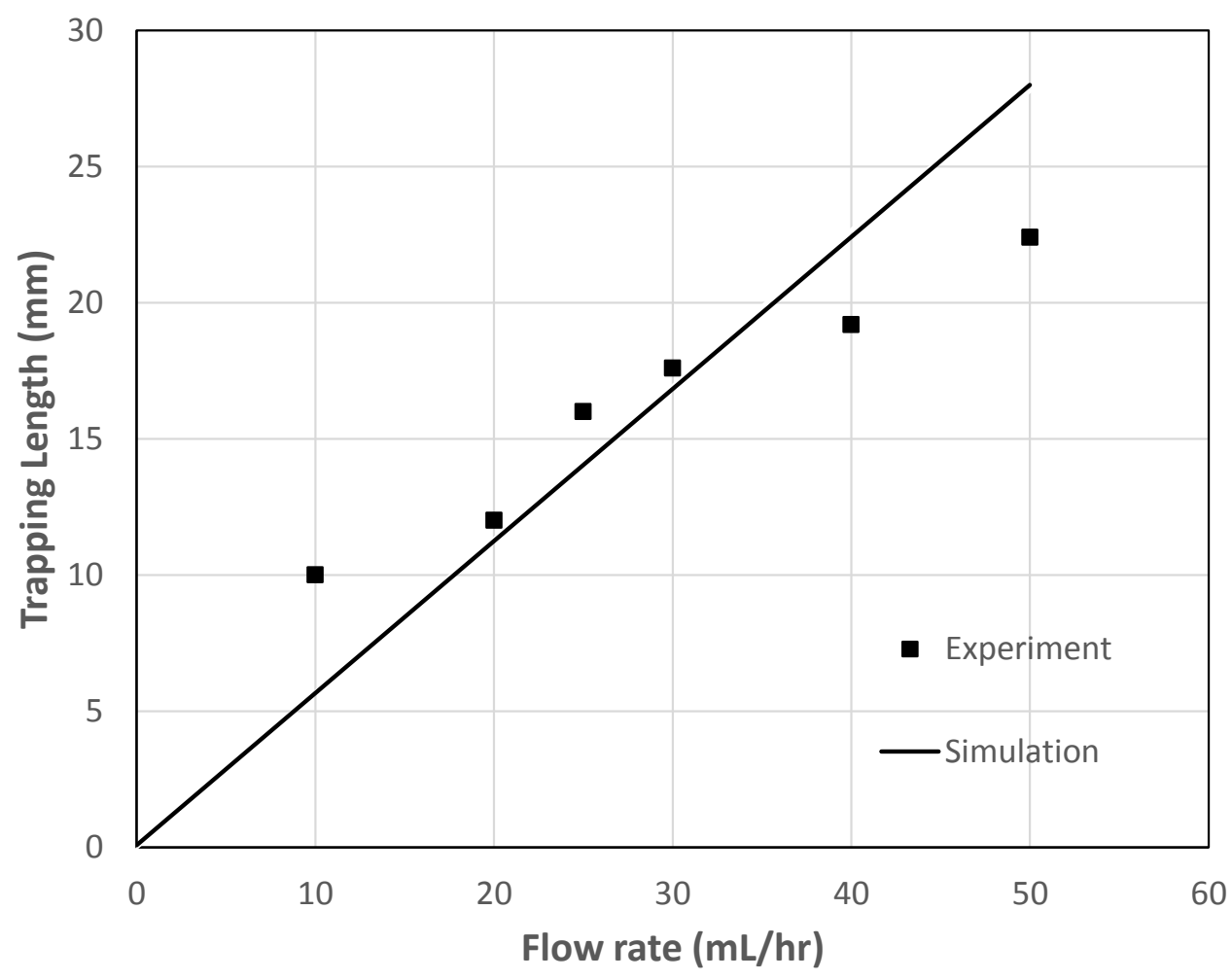

Figure 8 A comparison between the experimental and simulated trapping length at various flow rates

Figure 9 shows the cell trajectory at various numbers of magnetic beads per cell ranging from 2-10. The channel height was $100 \mu \mathrm{m}$, the cell size was $10 \mu \mathrm{m}$, the bead size was $1 \mu \mathrm{m}$, the substrate thickness was $300 \mu \mathrm{m}$, and the flow rate was $50 \mathrm{~mL} / \mathrm{h}$. The results indicate that the 
This is an author-created, un-copyedited version of an article published in Biomedical Microdevices. The final publication is available online at DOI 10.1007/s10544-016-0105-8

trapping length decreases with increasing the number of beads per cell. This is due to the fact that the magnetic force is proportional to the number of beads. Thus, a larger number of beads per cell result in a shorter trapping length. It should be noted that the fluid velocity near the top and bottom of the channel is smaller than the average velocity. This is the reason for the initial steep decrease in the vertical position of the cell as indicated by the concave region of the graphs. In addition, as the cell approaches the bottom of the microfluidic channel, the magnetic field gradient increases, resulting in an increase in the slope of the graph near the bottom of the channel. The effect of cell size on the particle trajectory is depicted in Fig. 10. Three different cell sizes of $8 \mu \mathrm{m}, 10 \mu \mathrm{m}$, and $12 \mu \mathrm{m}$ were simulated. The channel height was $100 \mu \mathrm{m}$, the bead size was $1 \mu \mathrm{m}$, the number of beads per cell was 6 , the substrate thickness was $300 \mu \mathrm{m}$, and the flow rate was $50 \mathrm{~mL} / \mathrm{h}$. The simulation results indicate that the trapping length increases as the cell size increases from $8 \mu \mathrm{m}$ to $12 \mu \mathrm{m}$. As shown in Eq. 8, the magnitude of the drag force is linearly proportional to the cell radius (i.e. the drag force is greater on larger particles). Thus, for the same magnetic force, larger cells move further downstream before being trapped, resulting in a larger tapping length.

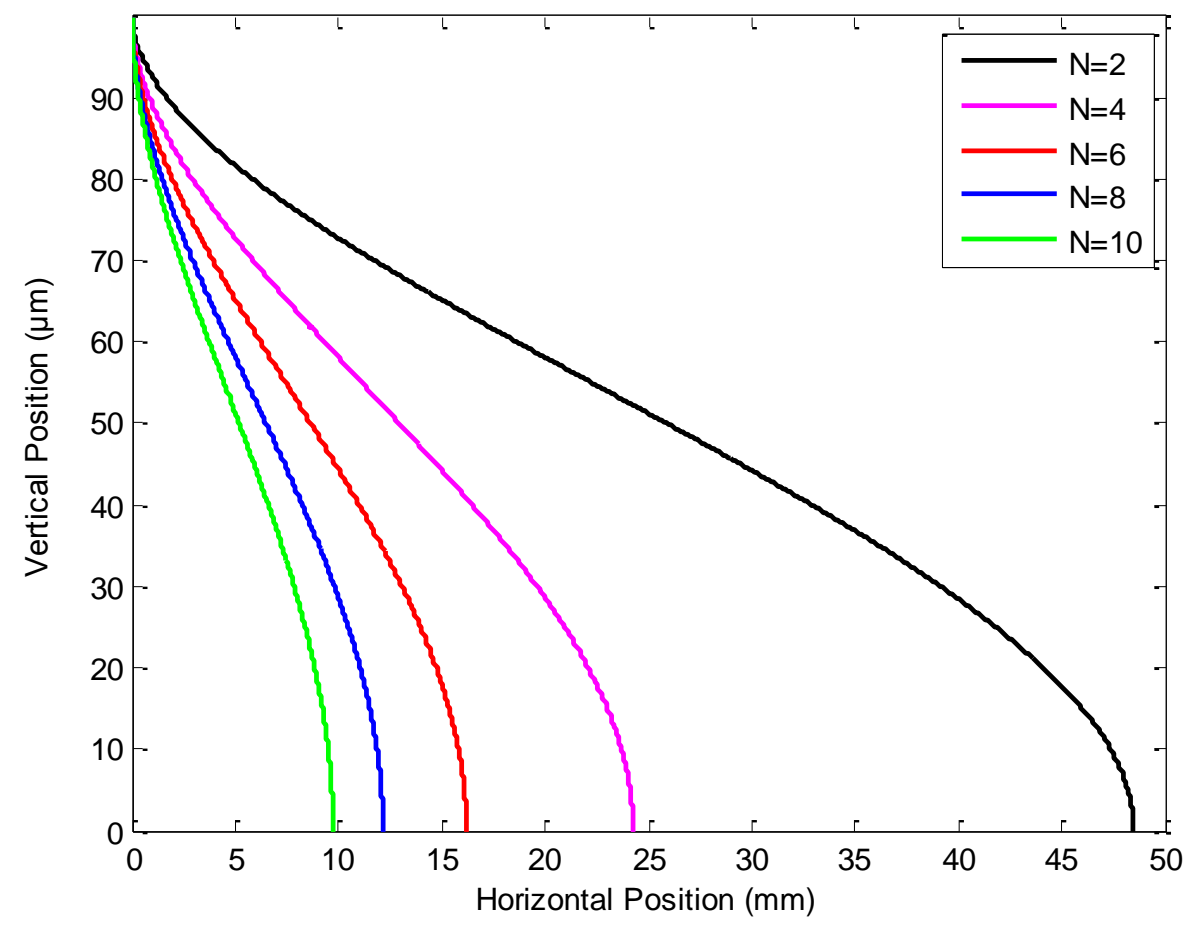

Figure 9 Cell trajectories along the microfluidic channel at various numbers of beads per cell. The cell size, bead size, channel height, substrate thickness, and flow rate were $10 \mu \mathrm{m}, 1 \mu \mathrm{m}$, $100 \mu \mathrm{m}, 300 \mu \mathrm{m}$, and $50 \mathrm{~mL} / \mathrm{h}$. 


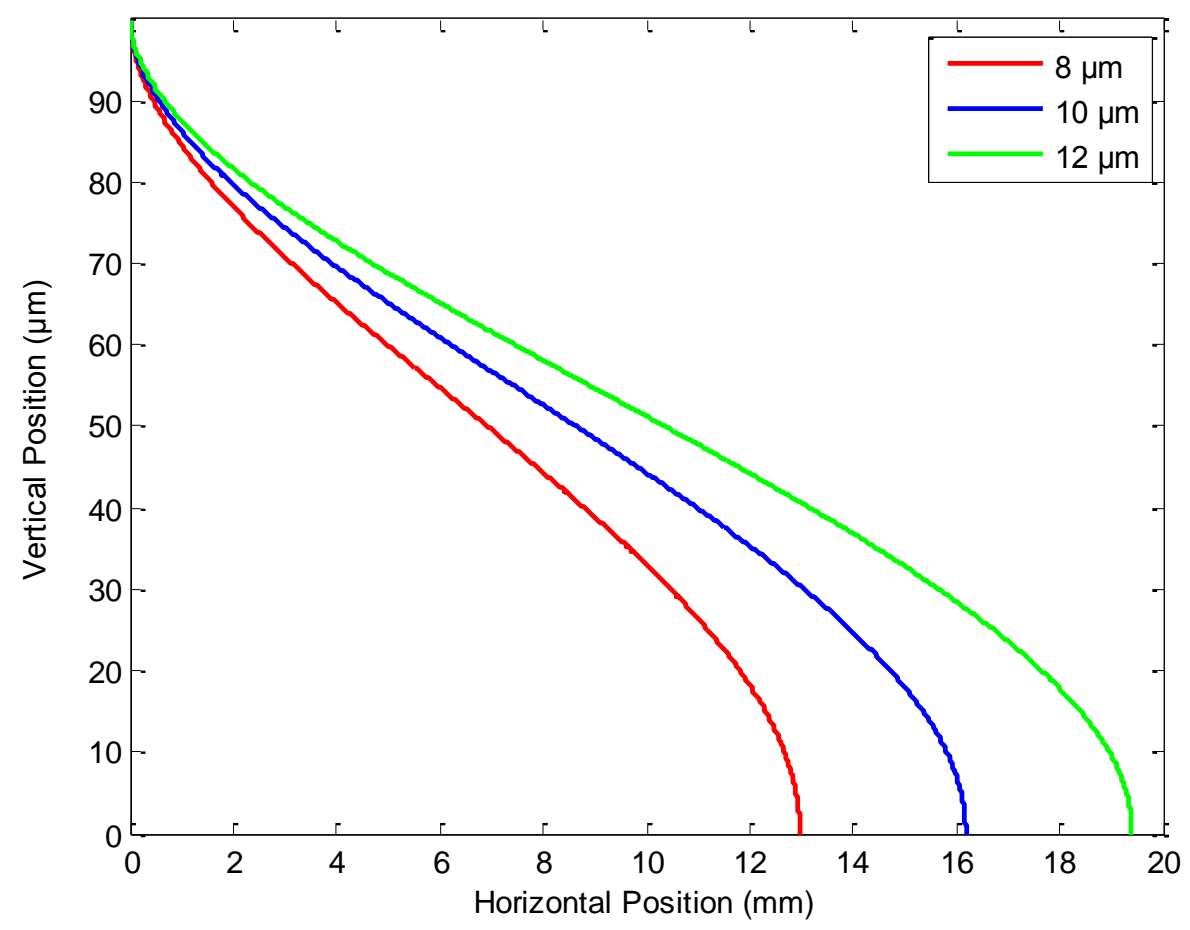

Figure 10 Cell trajectories at various cell sizes. The number of beads, bead size, channel height, substrate thickness, and flow rate were $6,1 \mu \mathrm{m}, 100 \mu \mathrm{m}, 300 \mu \mathrm{m}$, and $50 \mathrm{~mL} / \mathrm{h}$.

Figure 11 depicts the effect of the channel height on the trapping length. As seen there, the trapping length increases with increasing the channel height. This is because for a given sample flow rate, the magnitude of the magnetic field gradient decreases with increasing the channel height. Thus, the magnetic force is lower, which causes the cells to move further downstream before it is trapped. Figure 12 shows the effect of the substrate thickness on the trapping length. The results show that the trapping length increases with increasing the substrate thickness. This trend is similar to the channel height effect. Since the magnitude of the magnetic field gradient decreases with increasing the substrate thickness, the magnetic force decreases as well, resulting in an increase in the trapping length. The trajectory of the cell along the channel at various flow rates is shown in Fig. 13. The number of beads, bead size, cell size, channel height, and substrate thickness were $6,1 \mu \mathrm{m}, 10 \mu \mathrm{m}, 100 \mu \mathrm{m}$, and $300 \mu \mathrm{m}$. As the flow rate increases, the particle velocity increases as well in the x-direction. Therefore, depending on the magnetic force strength, the cell travels farther downstream or even may not be captured as the flow rate increases. 
This is an author-created, un-copyedited version of an article published in Biomedical Microdevices. The final publication is available online at DOI 10.1007/s10544-016-0105-8

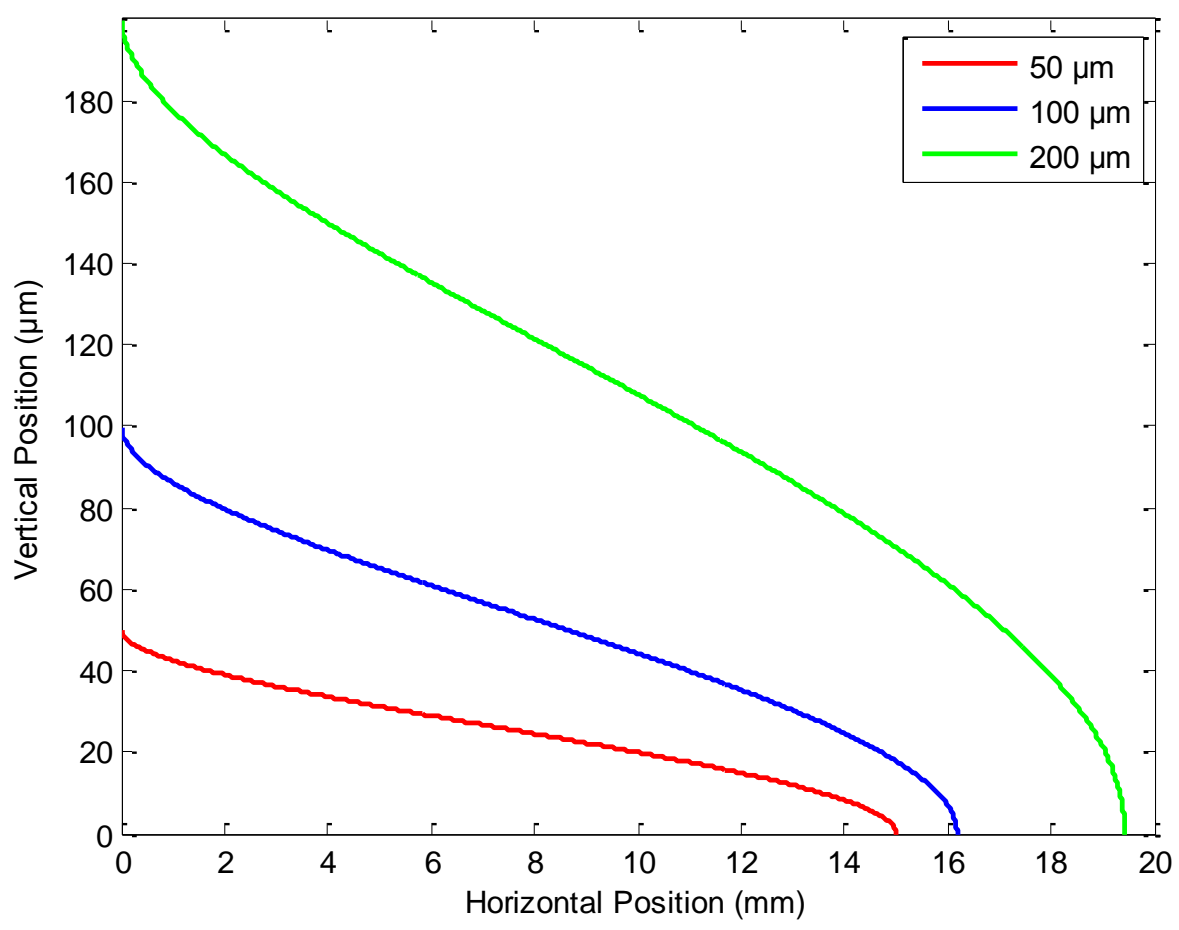

Figure 11 Cell trajectories at various channel heights. The number of bead, bead size, cell size, substrate thickness, and flow rate were $6,1 \mu \mathrm{m}, 10 \mu \mathrm{m}, 300 \mu \mathrm{m}$, and $50 \mathrm{~mL} / \mathrm{h}$.

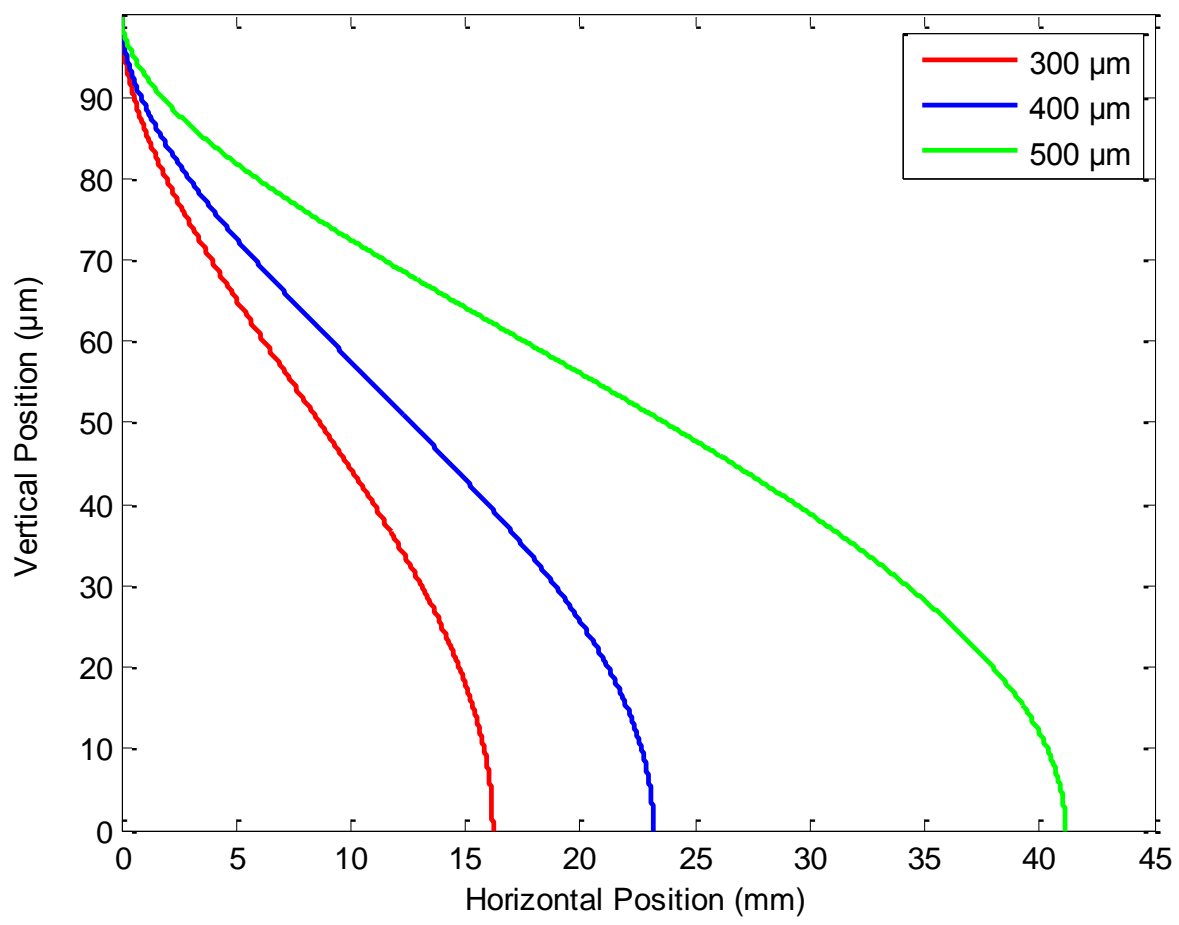

Figure 12 Cell trajectories at various substrate thicknesses. The number of beads, bead size, cell size, channel height, and flow rate were $6,1 \mu \mathrm{m}, 10 \mu \mathrm{m}, 100 \mu \mathrm{m}$, and $50 \mathrm{~mL} / \mathrm{h}$. 


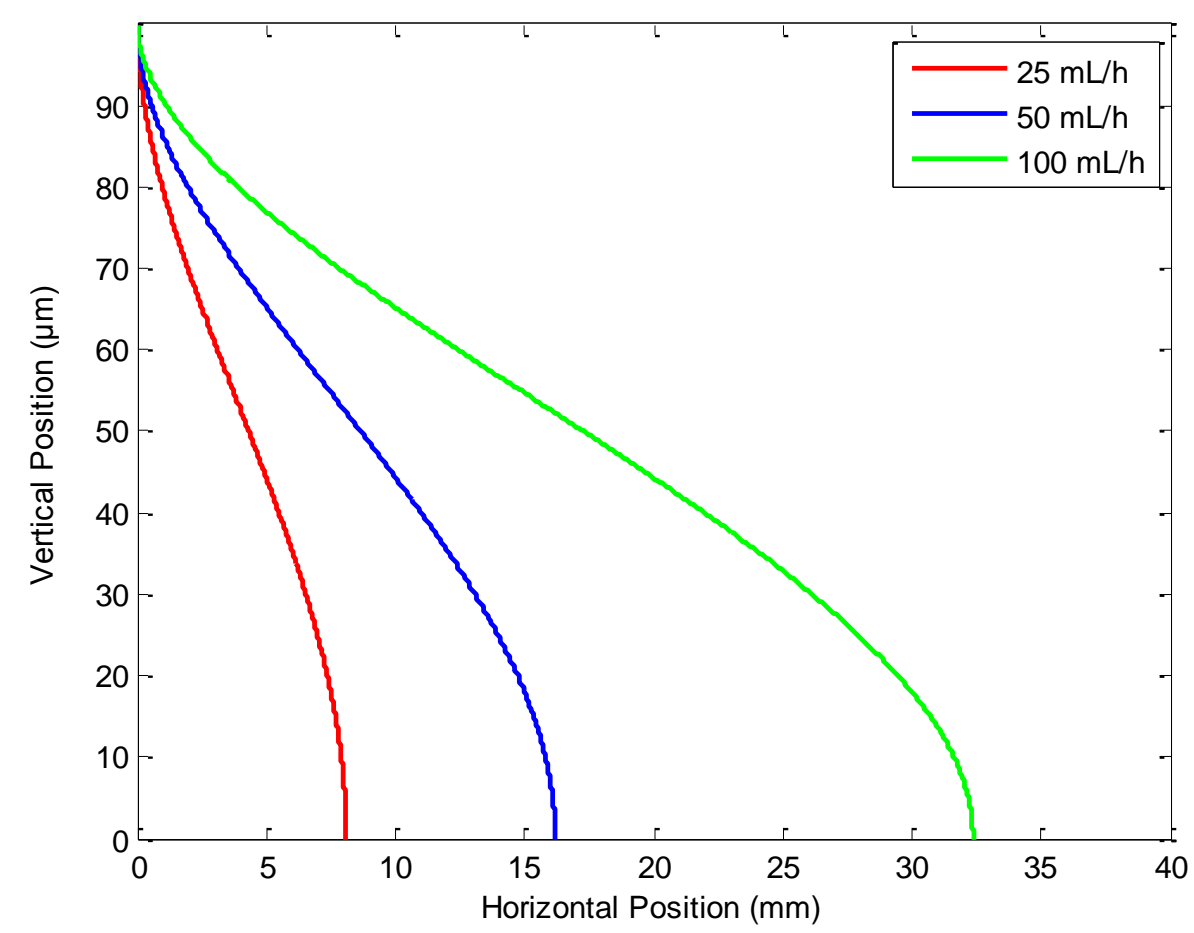

Figure 13 Cell trajectories at various flow rates. The number of beads, bead size, cell size, channel height, and substrate thickness were $6,1 \mu \mathrm{m}, 10 \mu \mathrm{m}, 100 \mu \mathrm{m}$, and $300 \mu \mathrm{m}$.

\section{Conclusions}

In this study, a microfluidic platform was successfully demonstrated for continuous isolation of highly purified human monocytes. The results indicate that a cell purity of nearly $90 \%$ and a recovery of $87 \%$ can be obtained at a flow rate of $50 \mathrm{~mL} / \mathrm{hr}$ in a single stage separation process. Additionally, a computational model was developed to simulate short- and long-range magnetic field gradients generated by and array of permanent magnets and micro-pattered nickel strips. The magnetic field simulations were then used to optimize the chip design and predict the particle motion and trajectory within the microfluidic channel. Furthermore, a comprehensive parametric study was conducted to investigate the effects of various geometric and operating parameters on the particle trajectory and trapping length. The trapping length was found to increase with increasing the cell size, channel height, substrate thickness, flow rate, while it decreased with increasing the number of beads per cell. The microfluidic device described here has the potential to be integrated into a Lab-on-a-Chip (LOC) system for rapid and effective separation of cells and molecules. 
This is an author-created, un-copyedited version of an article published in Biomedical Microdevices. The final publication is available online at DOI 10.1007/s10544-016-0105-8

\section{Acknowledgments}

This work was partially supported by a STEP grant from the SIUE Office of Research and Projects.

\section{References}

P. Campbell, Permanent Magnet Materials and Their Application, Cambridge University Press, 1996.

J. Darabi, C. Guo, On-chip magnetophoretic isolation of CD4+T cells from blood, Biomicrofluidics 7, 054106 doi: 10.1063/1.4821628, 2013.

E. P. Furlani and Y. Sahoo, Analytical model for the magnetic field and force in a magnetophoretic microsystem. J. Phys. D: Appl. Phys, 39, 1724-1732 (2006).

C. Hale and J. Darabi, Magnetophoretic-based microfluidic device for DNA isolation, Biomicrofluidics 8, 044118 (2014).

K. Hoshino at al., Microchip based Immunomagnetic Detection of Circulating Tumor Cell, Lab on Chip, 11, 3449-3457, 2011.

Y.Y. Huang et al., Screening and Molecular Analysis of Single Circulating Tumor Cells Using Micromagnet Array. Sci. Rep. 5, 16047; (2015).

N.M. Karabacak et al., Microfluidic, marker-free isolation of circulating tumor cells from blood samples, Nat Protoc. 2014 March ; 9(3): 694-710.

S. Kim et al., Circulating Tumor Cell Microseparator Based on Lateral Magnetophoresis and Immunomagnetic Nanobeads, Anal. Chem., 2013, 85 (5), pp 2779-2786.

A.J. Mach, D. Di Carlo, Continuous Scalable Blood Filtration Device Using Inertial Microfluidics, Biotechnol Bioeng. 2010, 107, 302-11.

H-S. Moon et al., Continuous separation of breast cancer cells from blood samples using multi-orifice flow fractionation (MOFF) and dielectrophoresis (DEP), Lab Chip, 2011,11, $1118-1125$ 
This is an author-created, un-copyedited version of an article published in Biomedical Microdevices. The final publication is available online at DOI 10.1007/s10544-016-0105-8

S. Nagrath et al., Isolation of rare circulating tumor cells in cancer patients by microchip technology, Nature 2007, 450, 1235-1239.

E. Ozkumur et al., Inertial Focusing for Tumor Antigen-Dependent and -Independent Sorting of Rare Circulating Tumor Cell, Sci Transl Med. 2013 April 3; 5(179).

J. W. Park et al., Microdevice for Separation of Circulating Tumor Cells Using Embedded Magnetophoresis with V-shaped Ni-Co Nanowires and Immuno-nanomagnetic Beads, vol. 37, no. 2, Apr. 2015, pp. 233-240.

E.M. Purcell, Electricity and Magnetism, 2nd Edition, McGraw-Hill, Inc., New York, NY, 1985.

B-Y. Qu et al., A glass microfluidic chip for continuous blood cell sorting by a magnetic gradient without labeling, Anal Bioanal Chem. 2008, 392, 1317-24 .

I. Safaryk and M. Safarykova, Scientific and Clinical Applications of Magnetic Carriers, ed. by U. Hafeli, W. Schutt, J. Teller, and M. Zborowski (Plenum press, New York, 1997).

S.S. Shevkoplyas et al., The force acting on a superparamagnetic bead due to an applied magnetic field, The Royal Society of Chemistry 2007, Lab Chip, 7, 1294-1302 (2007).

S.L. Stott et al., Isolation of circulating tumor cells using a microvortex-generating herringbonechip, Proc. Natl. Acad. Sci.USA, 2010; 107:1012539.

A.H. Talasaz et al., Isolating highly enriched populations of circulating epithelial cells and other rare cells from blood using a magnetic sweeper device, Proc. Natl. Acad. Sci. USA, 2009; 106:3970-3975.

N. Yuan et al., A method for rapid isolation of highly purified human monocytes using fully automated negative cell selection, The Journal of Immunology, 2007, 178, 36.25.

C-H. Wu et al., Versatile Immunomagnetic Nanocarrier Platform for Capturing Cancer Cells, ACS Nano, 10.1021/nn403281e, 2013.

M. Zborowski et al., Analytical magnetapheresis of ferritin-labeled lymphocytes, Anal. Chem, 67, 3702-3712 (1995). 